\title{
Portable High-End Instrument for In-Vivo Infrared Spectroscopy Using Spread Spectrum Modulation
}

\author{
Mario E. Giardini ${ }^{1}$, Stefano Trevisan ${ }^{1}$ \\ ${ }^{1}$ INFM-Laboratorio FiMed - LITA, Via F.1li Cervi, 93 - 20090 Segrate (MI), Italy \\ contact info: mario.giardini@fimed.litasegr.unimi.it - phone (+39) 0250330332
}

\begin{abstract}
Near Infrared Spectroscopy (NIRS) can be employed to monitor noninvasively and continuously local changes in hemodynamics and oxygenation of human tissues. In particular, the technique can be particularly useful for muscular functional monitoring during unattended physical activity. A portable NIRS research-grade acquisition system, dedicated to low-noise measurements during muscular exercise, is presented. A spreadspectrum multiplexing scheme significantly enhances system performance.

The resulting instrument is compact, lightweight and efficient. Preliminary tests on oxygen consumption during exercise and venous occlusion show excellent detectivity and time response.
\end{abstract}

Keywords - NIRS, Infrared spectroscopy, In-vivo, Portable, Multiplexing, Spread-spectrum

\section{INTRODUCTION}

From an optical point of view, biological tissue can be considered as an absorbing matrix in which a high number of inhomogeneities, which act as light scatterers, are present [1]. When collimated near infrared (NIR) light is injected into the tissue and the collimated transmission is observed, light losses are mostly due to scattering rather than to absorption [2]. In limiting a transmissive optical analysis to thin tissue regions, such as fingers or earlobes, on thick tissue the scattering provides a way of recovering part of the injected light from the injection surface. The spectral analysis of the backscattered light is, in the medical field, normally referred to as NIRS (Near InfraRed Spectroscopy) [3].

In the NIR region, the optical absorption is strongly dominated by hemoglobin, the blood constituent that carries oxygen. Smaller contributions to absorption are given by other cromophores, whose absorption spectrum may give information on tissue oxygen consumption [4]. Oxygen metabolism is most certainly a crucial problem in many fields of medical activity. Sport medicine, rehabilitative cardiology, intensive care, anesthesia, tumor detection, rely on some form of metabolic oxygen analysis. Using NIRS, the key issue of real-time non-invasive evaluation of blood content and oxygenation in a non-stationary condition, such as upon exercise or during respiratory manouvres, can be approached by non-invasive optical measurements.

The possibility of performing the measurement without hindering other patient activities could therefore open a practical way of accessing metabolic and hemodynamic information related to everyday life activities, to exercise, to infrequent events, or to hostile environments.

In strongly feeling the need for a portable, compact and rugged NIRS acquisition system, we require the instrument to present a number of measurement channels sufficiently high for a complete clinical picture even in demanding research applications. The large number of channels should be complemented by flexible input stages, in order to accomodate for different probe designs and for auxiliary nonNIRS signals. It should be able to operate reliably in a noncontrolled and non-conditioned environment. And, finally, it should provide for some sort of remote data transmission.

In the present work, a compact and portable instrument, dedicated to optical characterization of tissue is addressed. The instrument, which has been developed within the activities of the Italian National Institute for Physics of Matter - INFM - has been designed along the framework of the proprietary IRIS (InfraRed In-vivo Spectrometer) series $[5,6]$, and has been called IRIS-3.

\section{HARDWARE}

From a hardware point of view, IRIS-3 is composed by a battery-powered stand-alone portable unit, to which emitter/receiver probes are connected. Two source and two detector channels are provided. As in the literature most quantitative algorithms rely on the backscattering data at 4 different wavelengths [7], each source channel can drive up to 4 sources, for a total of 8 independent sources. For signal detection, two preamplified and filtered softwareconfigurable inputs are available. Two auxiliary nonamplified analog inputs are also accessible for connection to auxiliary sensors. The instrument derives its supply from a rechargeable NiMH battery with a duration in excess of 24 hours.

The core of the instrument consists in a microcontroller carrying an analog-to-digital converters (ADC), an universal synchronous-asynchronous serial transmitter-receiver (USART), and memory addressing peripherals.

The microcontroller is interfaced to the two light source connectors through two four-channel LED drivers. The sources are switched on with an appropriate sequence, as detailed in the following paragraphs, and the resulting transmitted light is detected and preamplified by two independent detector channels. The amplified signals are then fed to the microcontroller ADC. The data is demultiplexed 
and filtered digitally, and stored on a 1 MByte static RAM bank, backed by a disposable lithium battery.

The measured data can be downloaded to external mass storage by a serial bidirectional cable or monodirectional 433 $\mathrm{MHz}$ radio link. The serial connection is also employed to configure the input stages and to set up the measurement parameters.

A 4-key keyboard, 4-character alphanumeric LED display, capable of low-temperature operation, on the instrument front panel and a piezo beeper provide the elementary I/O features necessary for stand-alone operation.

The instrument is enclosed in a $145 \times 84 \times 35 \mathrm{~mm}$ plastic case with internal conductive shielding, and weighs $370 \mathrm{~g}$ (batteries included).

As the hardware capabilities allow for much more than the fixed protocols up to now available on literature NIRS units, the actual measurement is sequenced by user-defined programs written in a dedicated high-level definition language running on a real-time interpreter. An external user interface, implemented on a personal computer under a 32-bit Microsoft Windows environment, can be used to perform all the instrument setup, measurement protocol definition and debugging, configuration upload and data download functions.

The large flexibility in measurement protocol definition allowed by a user-defined acquisition sequence calls for equally flexible probe amplifier inputs, which should be adequate both for NIRS and for general detector input purposes. The two detector channels have therefore been equipped with high-performance filters-amplifiers with software-configurable coupling, gain and filtering actions. They can be configured as high-sensitivity transconductance amplifiers, for direct photodiode input, or as voltage followers, for preamplified probes. Moreover, one of them can be reconfigured as a differential amplifier with active shield driver capabilities, with adequate performance for the detection of the heartbeat signal, e.g. for heart rate analysis.

\section{MODULATION PROTOCOL}

In the most general case, when NIR light is injected into the tissue using $\mathrm{N}$ sources and a signal is detected on a suitable detector, the detection system must be able to demultiplex each response component to the corresponding source. For the best clinical significance of the NIRS data, the time lag between the signals from different sources and the total source scan cycle time should be minimized. It would be therefore desirable to be able to switch on all the sources simultaneously, integrate them and yet be able to distinguish the individual source contributions.

If $\mathrm{N}$ sources modulated using a set of $\mathrm{N}$ functions $\mathrm{f}_{\mathrm{n}}(\mathrm{n}=$ $1 \ldots \mathrm{N})$ with minimal cross-correlation are simultaneously applied to the tissue, and the NIRS signal is detected by a single detector, in the simplest case of a linear response with no delays, the detector signal $\mathrm{S}$ is constituted by the $\mathrm{N}$ source components as:

$$
S=\sum_{n=1 \ldots N} a_{n} f_{n}
$$

where $a_{n}$ are the response amplitudes associated to the single sources. In order to associate each component to the appropriate source, the signal can be multiplied independently by the modulation functions and timeintegrated. For the n-th source:

$$
\begin{aligned}
S_{n} & =\int f_{n} S d t \\
& =\sum_{m=1 . . N} \int a_{m} f_{n} f_{m} d t \\
& =a_{n} \int f_{n}^{2} d t
\end{aligned}
$$

as the modulation functions cross correlations vanish, and only the n-th term of the sum is non-null.

We note that, after the signal has been digitized, all the multiplication and integration can be performed digitally by simple multiply-accumulate operations. If the computing power of the processor is adequate, the actual hardware implementation can be therefore reduced to the single source drivers and to the detector preamplifier, as in our apparatus.

When the modulation functions are chosen as sinusoids or square waves with different frequencies (frequency multiplexing by lock-in detection [8]), in order to obtain an acceptable signal-to-noise ratio and low channel crosstalk in a purely digital implementation, the signal sampling period must be significantly lower and the integration time significantly higher than the sinusoid period. This may easily require, even for low sinusoid frequencies, fast $\mathrm{AD}$ converters and high processor clock rates [9].

A more subtle drawback of digital lock-in for NIRS is related to the fact that, if the modulation frequencies are kept in the $\mathrm{kHz}$ range in order to employ cost-effective technologies, they are quite too near to the frequency of common narrowband noise sources in a medical environment, i.e. ambient light, electrosurgical unit discharges and high-power switching-mode supplies, quite common in clean rooms or operating theatres. Moreover, for portable equipment, the patient moves in an environment that cannot always be fully characterized with respect to narrowspectrum electromagnetic interference.

Our choice of the modulation functions $\mathrm{f}_{\mathrm{n}}$ comes from the consideration that independent noise sources by definition show high autocorrelation and low cross-correlation. The $f_{n}$ can therefore be chosen as noise-like signals, presenting with respect to sinusoids the additional advantage that the only signal that could contaminate the demultiplexing of the $n$-th source is something similar to $f_{n}$ itself, and such a noise is extremely unlikely to occur. Such modulation, known as 
"spread spectrum" [10], is used in telecommunications mainly for data security reasons.

From our functional point of view, the attention has been focused on employing as $f_{n}$ functions (freely available in the literature) which oscillate between 0 (source off) and 1 (source on) states in a pseudo-random sequence with minimal cross-correlation, switching on multiples of a common time interval (i.e., we require the sequences to change state synchronously with a common clock). We also require the sequences to be periodic with a common period. These two requirements allow to precalculate the sequences in a look-up table for the microcontroller. The look-up table is interrogated (on the common clock tick) for all the sources simultaneously, the source states are set accordingly, and the detector sampling frequency can be as low as the common clock, thus significantly reducing costs and complexity of the $\mathrm{ADC}$ and of the required computing.

\section{RESULTS}

An example of a typical acquisition signal is reported in Fig. 1. The NIRS probe has been placed on a large leg muscle of a 28-year old male volunteer, and an incremental load exercise protocol (A) followed by tourniquet-induced venous occlusion (B) has been performed on a cyclette.

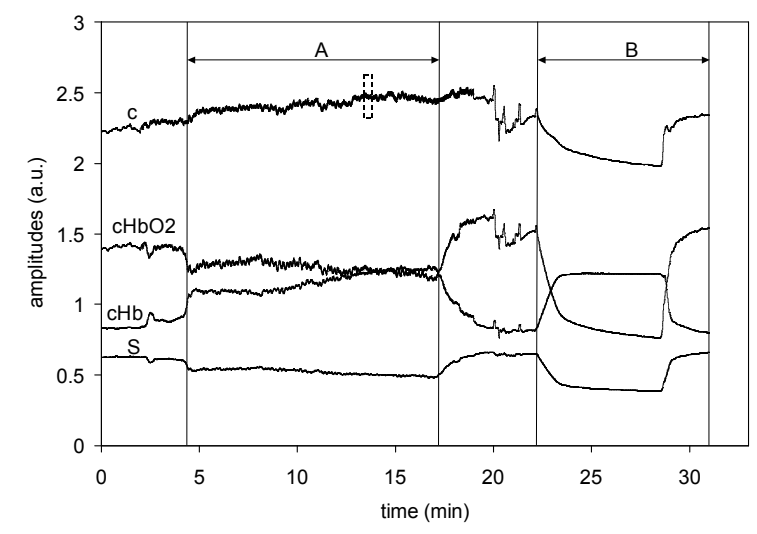

Fig. 1. Measurement during an incremental load exercise

Both in the incremental exercise (A) and in the venous occlusion (B) the total haemoglobin concentration (c), the oxidized $(\mathrm{HbO} 2)$ and reduced $(\mathrm{Hb})$ haemoglobin concentrations and the absolute tissue oxygen saturation (s) follow the expected behaviors. While a detailed description of the results goes beyond the scope of the present paper and, with the relevant figures, will be given in an extended version, we note good correspondence of the data with the known phenomenology [11,12].

Moreover, if we expand the time scale of the incremental exercise (A), we see that the signal fluctuation is actually associated with the variation of the measured parameters during the pedal cycle. In other terms, the signal to noise ratio is sufficiently high as to be able to detect the hemodynamic changes (blood content and oxygen saturation) associated with the single pedal cycles. As an example, in Fig. 2 is reported the expansion of the dashed rectangle in Fig. 1. The periodic behavior of the haemoglobin concentration can be clearly identified.

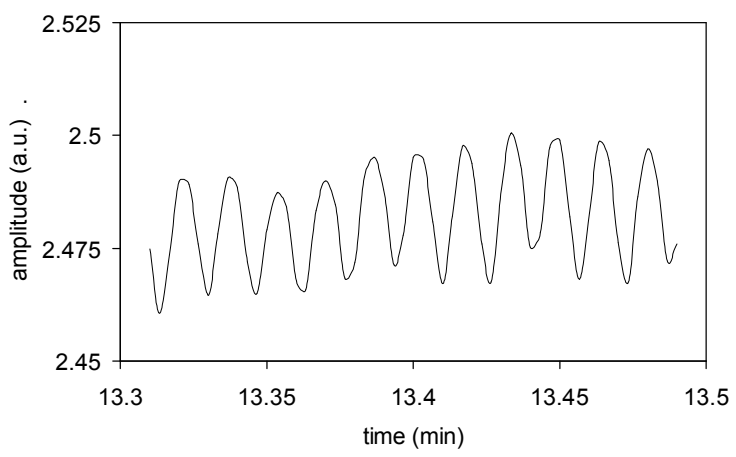

Fig. 2. Expansion of insert in Fig. 1

\section{CONCLUSIONS}

In all, these considerations have allowed the construction of a portable instrument up to the standards of the best continuous-wave fixed instruments in the literature, with a reduction in costs and complexity as significant as to be compatible for mass production of the instrument and its introduction in the common clinical practice.

\section{IPR COVERAGE}

The Intellectual Property Rights for the technologies described in this paper are covered by INFM, the Italian National Institute for Physics of Matter (www.infm.it), that should be contacted, directly or through the Authors, for any matter that may apply.

\section{ACKNOWLEDGEMENTS}

We wish to thank The Italian National Institute for Physics of Matter (INFM) for funding, Mario Corti (INFM) for useful discussion and Matteo Bavera for help in the design of the instrument prototypes.

\section{REFERENCES}

[1] V. Tuchin, Tissue Optics, SPIE, Bellingham (U.S.A), 2000

[2] F.F. Jöbsis, "Non-invasive, infra-red monitoring of cerebral and myocardial oxygen sufficiency and circulatory parameters", Science Vol. 198, pp. 1264-1267 (1977)

[3] H. Owen-Reece, M. Smith, C.E.Elwell and J.C. Goldstone, "Nearinfrared spectroscopy", Br J Anaesth Vol. 82 (3), pp. 418-426 (1999)

[4] S. Wray, M. Cope, D.T. Delpy and J.S. Wyatt, , and E.O.R. Reynolds, "Characterization of the near infrared absorption spectra of cytochrome aa3 and hemoglobin for the non-invasive monitoring of cerebral oxygenation”, Biochim Biophys Acta Vol. 933, pp. 184-192 (1988) 
[5] M.E.Giardini, M.Corti, G.G.Guizzetti, P.Lago, A.Gelmetti and G.Danese, "microcontroller-based digital front-end for near-infrared spectroscopy", SPIE Proc Vol. 3911, pp. 338-344 (2000)

[6] M.E.Giardini, M.Corti, P.Lago and A.Gelmetti, "portable microcontroller-based instrument for near infrared spectroscopy", SPIE Proc Vol. 3911, pp. 250-255 (2000)

[7] S.J. Matcher, C.E. Elwell, C.E.Cooper and D.T. Delpy, "Performance comparison of several published tissue near-infrared spectroscopy algorithms", Anal Biochem Vol. 227, pp. 54-68 (1995)

[8] M.L. Meade, Lock-in amplifiers: principles and applications, Peter Peregrinus Ltd., London (U.K.), 1983

[9] G.G.Guizzetti, G.Danese, F.Leporati and M.E.Giardini, "An intelligent acquisition system for near infrared spectroscopy", in Medical Diagnostic Techniques and Procedures, M.Singh et al. (eds), pp. 182187, Narosa Publishing House, New Delhi, India (1999)

[10] R.E. Ziemer, R.L. Peterson, D.E.Borth, Introduction to spreadspectrum communications, Prentice Hall Inc, Upper Saddle River (U.S.A), 1995

[11] B. Chance, S. Nioka, J. Kent, K. McCully, M. Fountain, R. Greenfeld and G. Holtom, "Time-Resolved Spectroscopy of Hemoglobin and Myoglobin in Resting and Ischemic Muscle", Anal Biochem Vol. 174, pp. 698-707 (1988)

[12] B. Cance, M.T. Dait, C. Zhang, T. Hamaoka and F. Hagerman, "Recovery from Exercise-induced Desaturation in the Quadriceps Muscles of Elite Competitive Rowers", Am J Physiol Vol. 262, pp. C776-775 (1992) 